\title{
Evaluation of Results of Anterior Cervicothoracic Junction Decompression \& Fusion (ACTJDF) of C7-T1-T2 Spinal Tuberculosis by Transmanubrial Transclavicular Approach
}

\author{
Atanu Mohanty ${ }^{1}$, Rupak Biswas², Anuraag Mohanty ${ }^{3}$ \\ ${ }^{1}$ Department of Orthopaedics, SCB Medical College, Cuttack, Odisha, India. ${ }^{2}$ Department of Orthopaedics, \\ SCB Medical College, Cuttack, Odisha, India. ${ }^{3}$ IMS and Sum Hospital, Bhubaneswar, Odisha, India.
}

\section{ABSTRACT}

\section{BACKGROUND}

Spinal tuberculosis is the most frequently encountered \& destructive form of skeletal tuberculosis. We wanted to evaluate the advantages of transmanubrial transclavicular approach in the surgical management of the pathologies (mainly tuberculosis) at the cervicothoracic junction (C7,T1-2).

\section{METHODS}

From June 2013 to August 2019, thirty patients with tuberculosis of CTJ presented to SCBMC \& $\mathrm{H}$ and were operated with transmanubrial transclavicular approach. Debridement with corpectomy of tuberculosis of C7-T1 and T1 vertebral bodies were followed by fusion of C6-T2 and C7-T2 vertebra respectively with the help of titanium mesh cage and anterior cervical plating. Advantages \& limitations of this surgical approach were evaluated retrospectively. Visual analogue scoring \& Frankel scoring were used for assessing postoperative clinical outcome.

\section{RESULTS}

All 30 cases were of tuberculosis of CTJ involving either C7-T1 or T1-T2. They presented with a permutation combination of upper thoracic pain, paraparesis, and collapse of vertebra \& cord compression. All patients were followed up for $12.37 \pm$ 5.38 months on average (range $4-24 \mathrm{~m}$ ). The mean postoperative visual analogue scale score was $7.86 \pm 1.02$ (range 6-10) preoperatively, which improved to $3.27 \pm$ 0.88 (range $0-5)$ postoperatively at final evaluation $(\mathrm{p}<0.005)$. There was also postoperative increase in Frankel score in seventeen cases (56\%). Three patients developed temporary hoarseness postoperatively, two cases got infected (one superficial \& one deep) \& one patient died. There was no non-union or persistent pain at manubrium or clavicle end.

\section{CONCLUSIONS}

The transmanubrial transclavicular approach for tuberculosis of CTJ lesions can achieve favourable clinical outcomes by providing wide exposure, direct decompression of lesions, fusions and less complications as compared to standard approaches.

\section{KEY WORDS}

CTJ (Cervico-Thoracic Junction), Tuberculosis, Corpectomy
Corresponding Author:

Dr. Atanu Mohanty,

Friends Colony,

Bajrakabati Raod,

Cuttack-753001,

Odisha, India.

E-mail: anuraag98@gmail.com

DOI: $10.14260 /$ jemds/2020/195

Financial or Other Competing Interests: None.

How to Cite This Article:

Mohanty A, Biswas R, Mohanty A. Evaluation of results of anterior cervicothoracic junction decompression \& fusion (ACTJDF) of C7-T1-T2 spinal tuberculosis by transmanubrial transclavicular approach. J. Evolution Med. Dent. Sci. 2020;9(12):905-908, DOI: 10.14260/jemds/2020/195

Submission 17-01-2019,

Peer Review 29-02-2020,

Acceptance 04-03-2020,

Published 23-03-2020.

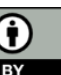




\section{BACKGROUND}

Though the first documented spinal tuberculosis (TB) case dates back to 5,000 years old Egyptian mummies, the first modern case of spinal TB was described by Percival Pott in 1779. Increasing frequency of $\mathrm{TB}$ in both developed \& developing countries has continued to make spinal TB a big health problem. Spinal tuberculosis, which is a common extrapulmonary variety, is the most frequent \& destructive form of skeletal tuberculosis. It accounts for approximately half of all cases of musculoskeletal tuberculosis. Although the thoracolumbar junction seems to be the most common site of spinal column involvement in spinal TB, any part of the spine can be affected. However, involvement of the cervico-thoracic junction is relatively uncommon, accounting for only $5 \%$ of total cases. ${ }^{1}$ It is more common in children \& young adults. Anatomically, the cervicothoracic junction is defined by most authors as an area extending from C7 to T4.It is a transitional zone between the lordotic cervical spine and the kyphotic thoracic spine. As the cervicothoracic vertebrae are weight bearing structures, so destruction of these vertebrae by TB frequently results in spinal instability, severe kyphotic deformity, large paravertebral abscesses \& progressive neurological deficit.

The potent anti-tubercular therapy (ATT) \& external immobilization are still corner stones for treatment of cervico-thoracic junction tuberculosis in most cases. But when it is characterized by bone destruction, severe kyphosis deformity, large abscess or spinal cord compression, surgical intervention is must along with ATT treatment to obtain a good result. Surgical intervention in such cases include decompression (e.g. corpectomy of effected vertebrae etc.), correction of kyphosis, reconstruction and stabilization in the form of fusion. Surgical approaches to cervico-thoracic tuberculosis includes anterior, posterior or combined anterior \& posterior approaches. Each approach has its own pros \& cons. The advantages of stand-alone anterior approach include improved visualization, radical debridement, directly addressing the clinically relevant pathology, more extensive decompression \& reconstruction of the anterior column, theoretically leading to improving rates of bone fusion \& getting perfect clinical outcome.

Cervico-thoracic spine tuberculosis that require decompression and stabilization need a wide anterior exposure involving at least one vertebral level above and one below the site. ${ }^{2}$ Of the entire spine, the upper thoracic spine is perhaps the least accessible and challenging due to the overlying clavicle, sternum and its close proximity of the great vessels. ${ }^{3}$ Nerves, lymphatics, and vascular structures which cross the upper mediastinum also complicate the operative exposure. An anterior cervical approach combined with median sternotomy for cervico-thoracic junction pathologies has been reported in literature.4,5 Later clavicle splitting was added with sternotomy for those pathologies. 6 This transmanubrial transclavicular approach has been popularized by Sundaresan et al, which was later on modified by others. ${ }^{7}$ The purpose of our prospective study was to evaluate the advantages of transmanubrial transclavicular (anterior) approach for decompression \& stabilization (fusion) in a randomized series of 30 patients with tuberculosis of cervico-thoracic junction vertebrae
(C7,T1-2) where surgical treatment was indicated along with ATT in respect of better exposure \& lower complication rate.

\section{METHODS}

\section{Patient Population and Evaluation}

After obtaining approval from the Medical Ethics Committee of SCBMC\&H, Cuttack for this study, informed consent was obtained from all the patients who presented with tuberculosis of CTJ to SCBMC\&H. All patients were provided written informed consent prior to participation. A randomized series of 30 patients with tuberculosis of CTJ presenting between June 2013 and august 2019 to SCBMC\&H who underwent anterior cervicothoracic junction decompression \& fusion (ACTJDF) by transmanubrial transclavicular approach were enrolled respectively in this study. Most of them presented with upper thoracic pain, weakness of lower limbs \& with or without difficulty in bowel bladder control. Thirteen of them gave a history of recent weight loss, constitutional symptoms or hemoptysis. Age, sex, lesion level, pathology, preoperative and postoperative neurological status and complications data were collected retrospectively. visual analogue scale (VAS) ${ }^{8}$ was used preoperatively and at 2 weeks postoperatively for pain scoring. To assess the pre and postoperative neurological status Frankel scores were used. The mean follow-up duration was 12.37 months (range $4 \mathrm{~m}-24 \mathrm{~m}$ ).

\section{Preoperative Evaluation \& Planning}

After proper clinical examination, routine blood tests, ESR, CRP, serum electrophoresis, PPD skin test, chest and CTJ $\mathrm{x}$ ray and sputum for AFB and malignant cells were done. Tuberculosis were confirmed by HPE via needle biopsy or by post-surgery tissue biopsy. Radiograph showed decreased height of C7 or T1 vertebral body depending upon the level of pathology. MRI revealed complete or partial destruction of $\mathrm{C} 7$ or $\mathrm{T} 1$ or both vertebral bodies with retropulsion of disc on to the cord. It also showed right or left sided paraspinal soft tissue mass and cord compression in all the cases. In view of ensuing cord compression with deteriorating neurological status, surgical debridement with fixation was planned. All the patients received antitubercular therapy for at least one month preoperatively, empirically or based on the results of needle biopsy.

\section{Operative Technique}

Under general anaesthesia patient was placed in supine position with a roll pad on the posterior of the back at the axis of spine which helped to keep both the shoulders in extended position. After routine surgical field preparation, a $\mathrm{T}$ shaped skin incision was made on left side of neck from medial lower $1 / 3^{\text {rd }}$ of sternocleidomastoid muscle up to manubrium sterni. Horizontal limb of $\mathrm{T}(1 \mathrm{~cm})$ above the clavicle \& the vertical limb extending in the midline over the body of the sternum. Elevation of medial $1 / 3^{\text {rd }}$ of left clavicle and left half of manubrium was done. The avascular tissue plane between the carotid sheath laterally and the trachea and esophagus medially was developed to reach the prevertebral space. Elevation of medial. 1/3rd of left clavicle and left half of manubrium was done. Debridement with corpectomy of C7-T1 and T1 vertebral bodies were followed 
by fusion of C6-T2 and C7-T2 vertebra with the help of titanium mesh cage and anterior cervical plating (GESCO, INDIA SYSTEM) for lesions effecting C7-T1 and T1-T2 respectively. Interestingly the cases of $\mathrm{C} 7-\mathrm{T} 1 \mathrm{~TB}$ spine showed destruction of C7 \& T1 vertebra for which C6 to T2 fixation was done whereas all the cases of T1-T2 TB spine had got only destruction of T1 body for which C7-T2 fusion was planned. Postoperatively antitubercular therapy was received by all the patients for $1 \mathrm{yr}$. as per protocol.

\section{RESULTS}

The mean age of the patients was $35.53 \pm 12.95$ years (range 13-65 years). Seventeen out of thirty patients were females $(57 \%)$. All of them presented with upper thoracic pain and 23 out of $30(76 \%)$ showed signs of progressive myelopathy. 16 patients (53\%) had involvement of C7-T1 vertebra and rest (47\%) of them had T1-T2 involvement (Table no 2). In all cases debridement with corpectomy of tuberculosis of C7-T1 and $\mathrm{T} 1$ vertebral bodies were followed by fusion of C6-T2 and C7-T2 vertebra respectively with the help of titanium mesh cage and anterior cervical plating via transmanubrial transclavicular approach. The mean follow-up period was $12.37 \pm 5.38$ months (range 4 to 24 months) (Table-1).

\section{Operational Parameters}

Average operative time was $72 \pm 12.90 \mathrm{~min}$ (range 60 to 90 min). Mean blood loss was $111.66 \pm 15.72 \mathrm{ml}$ (range 100 to $150 \mathrm{ml}$ ) (Table-1). No injury of the major vessels or the spinal cord was observed. None of the patients required postoperative blood transfusion.

\section{Clinical Outcomes}

Preoperative local \& upper thoracic radiating pain decreased in all cases. The mean preoperative VAS score was $7.86 \pm 1.02$ (range 6-10) which improved to $3.27 \pm 0.88$ (range 2-5) which was statistically significant $(\mathrm{p}<0.005) \quad($ Table-1). Frankel score increased in 17 cases $(56 \%)$ post operatively and in 12 cases $(40 \%)$ it remained unchanged.

\section{Radiological Outcome}

Most patients (27/30) achieved bony fusion at 6 to 9 months except two cases with multidrug resistant TB spondylitis. During the follow up, no instrumentation loosening, migration or breakage was observed. There was no nonunion or persistent pain at manubrium or clavicle end.

\section{Blood Parameters}

Except two multidrug resistant TB cases (6\%), in rest all cases ESR \& CRP returned to normal within 3 months of follow-up.

\section{Complications}

Three patients with tubercular lesion at T1-T2 developed temporary hoarseness postoperatively. Hoarseness resolved in all the three cases within several weeks after administering steroids. Postoperative infection was found in 2 cases, one superficial \& the other deep. The case with deep infection was debrided thoroughly and systemic antibiotics were continued for $1 \mathrm{wk}$. according to culture sensitivity report. It healed nicely with in 10 to 14 days. Death of one patient happened immediate postoperatively, probably due to associated medical comorbidities.

\section{Statistical Analysis}

SPSS software (ver. 19.0; SPSS Inc., USA) \& Microsoft Excel was used for all data analysis. All data has been presented in the form of mean, standard deviation and range. The difference between pre \& post-operative visual analogue score was evaluated using regression test for calculating $p$ value. A p value $<0.05$ was considered statistically significant.

\begin{tabular}{|ccc|}
\hline Parameters & Mean & Standard Deviation \\
1. Age (yrs.) & 35.53 & 12.95 \\
2. Operation time(min) & 72 & 12.90 \\
3. Blood loss(ml) & 111.66 & 15.72 \\
4. Pre op VAS & 7.86 & 1.02 \\
5. Post op VAS & 3.27 & 0.88 \\
6. Follow up (months) & 12.37 & 5.38 \\
\hline Table 1. Patient Demographic, Operative \& Postoperative Parameters \\
Expressed as Mean and Standard Deviation \\
\hline \multicolumn{3}{|c|}{ Parameters } \\
1. Male/ Female & Cases (Percentage) \\
2. C7-T1 TB cases & $16 \%$ / $(53 \%)$ \\
3. T1-T2 TB cases & $14(47 \%)$ \\
5. No. Of complications & $23(76 \%)$ \\
4. Progressive myelopathy on presentation & $6(20 \%)$ \\
6. Improved Frankel score (post op) & $17(56 \%)$ \\
7. Unchanged Frankel score (post op) & $12(40 \%)$ \\
8. Bony union at 9 m & $27(90 \%)$ \\
\hline Table 2. Patient Demographic, Complications \& Postoperative \\
Follow-Up Parameters Expressed as Percentages
\end{tabular}

\section{DISCUSSION}

TB is a common disease even today in the developing world. The incidence of extrapulmonary TB is 3\%, among which $10 \%$ of cases are skeletal TB. Spinal TB cases constitute $50 \%$ of skeletal tubercular infections. Dorsal spine is the commonest site for spinal tuberculosis. However involvement of the cervico-thoracic junction is relatively uncommon, accounting for $5 \%$ of total spinal TB cases. ${ }^{1}$ Though anti TB medications to a great extent have ameliorated the complications but still a significant percentage of patients of spinal tuberculosis present with late onset neurological deficit, instability \& kyphotic deformity. Prevention of these complications can be done by anterior cervicothoracic junction decompression \& fusion at early stage of presentation.

Approach to areas of the spine like the cervicothoracic junction is a surgical challenge. Direct access to the cervicothoracic area is generally difficult unless the patient has a long slender neck and drooping shoulders. ${ }^{9}$ The change from lordosis to kyphosis restricts the anterior approach and there are many vital structures to be managed during the operation. To reach the upper thoracic vertebrae a number of extensive approaches combining thoracotomy, sternotomy or clavicle resection with anterior dissection at the upper mediastinum have been proposed. Geiger et al ${ }^{10}$ claimed that with an oblique $5 \mathrm{~cm}$ incision parallel to the medial border of the sternocleidomastoid muscle it is possible to reach the lesions extending to T3 vertebra. This approach may be used for simple discectomy and interbody fusion at C7-Tl or for biopsy. But for the lesions to be excised followed by stabilization in the cervicothoracic region 
neither this approach nor the approach described by Cloward provide a good exposure. ${ }^{11}$ Although splitting the sternum provides some access to the lesion, exposure is still limited by sternocleidomastoid muscles and the clavicles. Partial resection of the clavicle and manubrium allows good access to the upper thoracic spine This approach allows access not only to the axial skeleton but also to the brachial plexus and related vessels. ${ }^{12}$ An HS, Vaccaro A et al agree that the techniques of resecting manubrium and clavicle are superior to their technique because the morbidity and mortality rates seem to be less ${ }^{13}$ There seem to be another benefit of this technique as it allows the supporting muscles of respiration, (sternocleidomastoid muscle) to remain firmly attached to the clavicle which prevents potential impairment of pulmonary function in patients with respiratory problems. In our study of 30 cases we had an average operative time of only hrs. 15 mins, average blood loss of $110 \mathrm{ml}$ and minimal postoperative complications.

\section{CONCLUSIONS}

We feel that as standard approaches to the cervicothoracic junction cannot provide a good exposure, transmanubrial transclavicular approach is a better alternative in terms of good exposure with lower complications specifically in cases that need wide exposure like surgeries of anterior cervicothoracic decompression and fusion for spinal tuberculosis.

\section{REFERENCES}

[1] Tuli SM. Tuberculosis of the skeletal system. $2^{\text {nd }}$ edn. New Delhi: Jaypee Brothers Publishers 1997: p. 181.
[2] Vaccaro A, An HS. Anterior exposures of the cervicothoracic junction. In: An HS, Riley L, eds. Atlas of surgery of the spine. London: Martin Dunitz Ltd., 1998: p. 113-30.

[3] Comey CH, McLaughlin MR, Moossy J. Anterior thoracic corpectomy without sternotomy: a strategy for the malignant disease of the upper thoracic spine. Acta Neurochir (Wein) 1997;139(8):712-8.

[4] Cauchoix J, Binet JP. Anterior surgical approaches to the spine. Ann R Coll Surg Engl 1957;21(4):234-43.

[5] Hodgson AR, Stock FE, Fang HS, et al. Anterior spinal fusion. The operative approach and pathological findings in 412 patients with Pott's disease of the spine. Br J Surg 1960;48(208):172-8.

[6] Standefer M, Hardy RW, Marks K, et al. Chondromyxoid fibroma of the cervical spine. A case report with a review of the literature and description of an operative approach to the lower anterior cervical spine. Neurosurgery 1982;11(2):288-92.

[7] Sundaresan N, Shah J, Feghali JG. A transsternal approach to the upper thoracic vertebrae. Am J Surg 1984;148(4):473-7.

[8] Huskisson EC. Measurement of pain. Lancet 1974;2(7889):1127-31.

[9] Charles R, Govender S. Anterior approach to the thoracic vertebrae. J Bone Joint Surg 1989;71(1):81-4.

[10] Geiger M, Roth PA, Wu JK. The anterior cervical approach to the cervicothoracic junction. Neurosurgery 1995;37(4):704-10.

[11] Cloward RB. The anterior approach for removal of ruptured cervical disks. J Neurosurg 1958;15(6):60217.

[12] Birch R, Bonney G, Marshall RW. Surgical approach to the cervicothoracic spine. J Bone Joint Surg $\mathrm{Br}$ 1990;72(5):904-7.

[13] An HS, Vaccaro A, Cotler JM, et al. Spinal disorders at the cervicothoracic junction. Spine 1994;19(22):2557-64. 